\title{
Semiclassical theory of spin-orbit interactions using spin coherent states
}

\author{
M. Pletyukhov ${ }^{1}$, Ch. Amann, M. Mehta ${ }^{2}$ and M. Brack \\ Institut für Theoretische Physik, Universität Regensburg, D-93040 Regensburg, Germany \\ ${ }^{1}$ e-mail: mikhail.pletyukhov@physik.uni-regensburg.de \\ ${ }^{2}$ present address: Harish-Chandra Research Institute, Chhatnag Road, Jhusi, Allahabad 211019, India
}

(November 3, 2018)

We formulate a semiclassical theory for systems with spinorbit interactions. Using spin coherent states, we start from the path integral in an extended phase space, formulate the classical dynamics of the coupled orbital and spin degrees of freedom, and calculate the ingredients of Gutzwiller's trace formula for the density of states. For a two-dimensional quantum dot with a spin-orbit interaction of Rashba type, we obtain satisfactory agreement with fully quantum-mechanical calculations. The mode-conversion problem, which arose in an earlier semiclassical approach, has hereby been overcome.

03.65.Sq,71.70.Ej,73.20.Dx,75.10.Hk

The incorporation of spin degrees of freedom in the semiclassical treatment of quantum systems represents a considerable challenge to the theorist. The periodic orbit theory (POT), initiated by Gutzwiller through his semiclassical trace formula [1], has been very successful in promoting the research on quantum chaos [2] and in explaining prominent quantum shell effects occurring in finite fermion systems in many domains of physics (see, e.g., [3]). The growing interest in "spintronics", i.e., the spin-polarized transport and spin dynamics in various electronic materials [ [I], with the particular scope of developing a spin transistor [5], make it desirable to formulate also the semiclassical theory including spin.

Littlejohn and Flynn have extended the POT for systems with multi-component wavefunctions and used it for the WKB quantization of spherical systems with spinorbit interactions [6]. They did not give an explicit trace formula, however, the main obstacle being the problem of "mode conversion" which arises when the spin-orbit interaction in phase space locally becomes zero. Frisk and Guhr [7] have applied the method of [6] to deformed cavities and promoted the hypothesis that spin-flips occur at the mode-conversion points through diabatic transitions between the two adiabatic spin-polarized energy surfaces. Bolte and Keppeler [8] have derived a relativistic trace formula from the Dirac equation and studied nonrelativistic limits, thereby justifying some ad hoc assumptions made in [7]. Their approach works well in the weak-coupling limit where the spin does not affect the orbital motion [9]. It may not be extensible [10], however, to strong spin-orbit interactions such as that observed in $p$-InAs or in InGaAs-InAlAs heterostructures [11], or also that amongst the nucleons in atomic nuclei [12]. For such systems, the approach presented below provides a semiclassical theory with a larger range of validity.
In this letter, we report on a semiclassical approach that allows for the explicit coupling between spin and orbital dynamics. We use the spin coherent state method and the path integral in an extended phase space to derive classical equations of motion which include the spin degrees of freedom. We then obtain the amplitudes in the semiclassical trace formula for the density of states without encountering the mode-conversion problem.

The path integral for a system with spin in the $\mathrm{SU}(2)$ spin coherent state representation originally appeared in a paper by Klauder [13] as an integral on $S^{2}$. Kuratsuji et al. 14. have represented it as an integral over paths in the extended complex plane $\bar{C}^{1}$. From the group theoretical point of view, the $\mathrm{SU}(2)$ coherent state can be associated with the unitary irreducible representation of the $\mathrm{SU}(2)$ group 15. To present its construction, we follow a recent paper by Kochetov [16] and his notations. The coherent state $|z, S\rangle$ for spin $S$ is defined by

$$
\begin{aligned}
& |z ; S\rangle=\left(1+|z|^{2}\right)^{-S} \exp \left(z \hat{s}_{+}\right)|S,-S\rangle, \\
& \hat{s}_{-}|S,-S\rangle=0
\end{aligned}
$$

where $z \in \bar{C}^{1}$ is a complex number, and $\hat{s}_{ \pm}=\hat{s}_{1} \pm i \hat{s}_{2}$, and $\hat{s}_{3}$ are the generators of the spin $\mathrm{SU}(2)$ algebra:

$$
\left[\hat{s}_{3}, \hat{s}_{ \pm}\right]= \pm \hat{s}_{ \pm}, \quad\left[\hat{s}_{+}, \hat{s}_{-}\right]=2 \hat{s}_{3} .
$$

$S \in N / 2$ denotes the representation index.

The irreducibility as well as the existence of the group invariant de Haar measure $d \mu_{S}$ ensures that the resolution of unity holds in the spin coherent state basis:

$$
\begin{gathered}
\int|z ; S\rangle\langle z ; S| d \mu_{S}(z)=I_{2 S+1}, \\
d \mu_{S}(z)=\frac{2 S+1}{\pi\left(1+|z|^{2}\right)^{2}} d^{2} z
\end{gathered}
$$

which turns out to be the most important property of spin coherent states that allows for the path integral construction, whereby the measure $d \mu_{S}$ takes account of the curvature of the sphere $S^{2}$. In what follows, we denote $|z ; S\rangle$ simply by $|z\rangle$ and use $z=u-i v$ with $u, v \in R^{1}$.

Let us now consider a quantum Hamiltonian with spinorbit interaction

$$
\widehat{H}=\widehat{H}_{0}(\hat{\mathbf{q}}, \hat{\mathbf{p}})+\kappa \hbar \hat{\mathbf{s}} \cdot \widehat{\mathbf{C}}(\hat{\mathbf{q}}, \hat{\mathbf{p}}),
$$

where $\hat{\mathbf{s}}=\left(\hat{s}_{1}, \hat{s}_{2}, \hat{s}_{3}\right)$. Hereby $\widehat{\mathbf{C}}=\left(\widehat{C}_{1}, \widehat{C}_{2}, \widehat{C}_{3}\right)$ is a vector function of the coordinate and momentum operators $\hat{\mathbf{q}}, \hat{\mathbf{p}}$, and the parameter $\kappa$ regulates the strength of the interaction. We make use of Smirnov's idea [17] to write 
the expression for the respective quantum propagator in terms of a path integral in both the orbital variables $\mathbf{q}, \mathbf{p}$ and the spin coherent state variables $v, u$. Imposing periodic boundary conditions on the propagation and thus integrating over closed paths, we arrive at the expression for the partition function (or trace of the propagator):

$$
Z(T)=\int \mathcal{D}[\mathbf{q}] \mathcal{D}[\mathbf{p}] \mathcal{D} \mu_{S}[z] \exp \{i \mathcal{R}[\mathbf{q}, \mathbf{p}, z ; T] / \hbar\},
$$

where $\mathcal{R}$ is Hamilton's principal action function, calculated along closed paths over a time interval $T$

$$
\begin{array}{r}
\mathcal{R}[\mathbf{q}, \mathbf{p}, z ; T]=\oint_{0}^{T}\left[\frac{1}{2}(\mathbf{p} \cdot \dot{\mathbf{q}}-\mathbf{q} \cdot \dot{\mathbf{p}})+2 S \hbar \frac{(u \dot{v}-v \dot{u})}{\left(1+|z|^{2}\right)}\right. \\
-\mathcal{H}(\mathbf{q}, \mathbf{p}, v, u)] d t
\end{array}
$$

and the path integration in (6) is taken over the $2(d+1)$ dimensional extended phase space:

$$
\mathcal{D}[\mathbf{q}] \mathcal{D}[\mathbf{p}] \mathcal{D} \mu_{S}[z]=\lim _{N \rightarrow \infty} \prod_{k=1}^{N} \prod_{j=1}^{d} \frac{d q_{j}\left(t_{k}\right) d p_{j}\left(t_{k}\right)}{2 \pi \hbar} d \mu_{S}\left(z_{k}\right) .
$$

Hereby the time interval $T$ is divided into $N$ time steps $t_{k}=k T / N$, and $z_{k}=z\left(t_{k}\right)$. Note that the periodic boundary conditions enable the antisymmetrization of the orbital part of the symplectic 1-form in (7).

The classical phase-space symbol $\mathcal{H}(\mathbf{q}, \mathbf{p}, v, u)$ of the Hamiltonian (5), appearing in the integrand of (7), is

$$
\mathcal{H}(\mathbf{q}, \mathbf{p}, v, u)=\mathcal{H}_{0}(\mathbf{q}, \mathbf{p})+\kappa \hbar S \mathbf{n}(v, u) \cdot \mathbf{C}(\mathbf{q}, \mathbf{p}),
$$

where $\mathcal{H}_{0}(\mathbf{q}, \mathbf{p})$ and $\mathbf{C}(\mathbf{q}, \mathbf{p})$ are the Wigner symbols of the operators $\widehat{H}_{0}$ and $\widehat{\mathbf{C}}$, and $\mathbf{n}=\left(n_{1}, n_{2}, n_{3}\right)=\langle z|\hat{\mathbf{s}}| z\rangle / S$ is the unit vector of dimensionless classical spin components given in terms of $u$ and $v$ by $n_{1}=2 u /\left(1+|z|^{2}\right)$, $n_{2}=2 v /\left(1+|z|^{2}\right)$, and $n_{3}=-\left(1-|z|^{2}\right) /\left(1+|z|^{2}\right)$.

The path integral (6) receives its largest contributions from the neighborhood of the classical paths along which the principal function $\mathcal{R}$ is stationary according to Hamilton's variational principle $\delta \mathcal{R}=0$. The first variation hereby yields the following equations of motion

$$
\begin{aligned}
& \dot{v}=\frac{\left(1+|z|^{2}\right)^{2}}{4 \hbar S} \frac{\partial \mathcal{H}}{\partial u}=\kappa \frac{\left(1+|z|^{2}\right)^{2}}{4} \frac{\partial \mathbf{n}(v, u)}{\partial u} \cdot \mathbf{C}(\mathbf{q}, \mathbf{p}) \\
& \dot{u}=-\frac{\left(1+|z|^{2}\right)^{2}}{4 \hbar S} \frac{\partial \mathcal{H}}{\partial v}=-\kappa \frac{\left(1+|z|^{2}\right)^{2}}{4} \frac{\partial \mathbf{n}(v, u)}{\partial v} \cdot \mathbf{C}(\mathbf{q}, \mathbf{p}) \\
& \dot{q}_{i}=\frac{\partial \mathcal{H}}{\partial p_{i}}=\frac{\partial \mathcal{H}_{0}}{\partial p_{i}}+\kappa \hbar S \mathbf{n}(v, u) \cdot \frac{\partial \mathbf{C}(\mathbf{q}, \mathbf{p})}{\partial p_{i}} \\
& \dot{p}_{i}=-\frac{\partial \mathcal{H}}{\partial q_{i}}=-\frac{\partial \mathcal{H}_{0}}{\partial q_{i}}-\kappa \hbar S \mathbf{n}(v, u) \cdot \frac{\partial \mathbf{C}(\mathbf{q}, \mathbf{p})}{\partial q_{i}}
\end{aligned}
$$

whose solutions define the classical orbits in the extended phase space. Note the appearance of $\hbar$ multiplying $S$ everywhere in (9), which reflects the non-classical nature of the spin. The two equations for the spin variables $v, u$ are equivalent to $\dot{\mathbf{n}}=-\kappa \mathbf{n} \times \mathbf{C}$, with the constraint $\mathbf{n}^{2}=1$. For spin-boson coupling in the Jaynes-Cummings model, equations analogous to (9) have been derived in [18].

Sugita 19] has recently given a re-derivation of Gutzwiller's trace formula, starting from $Z(T)$ whose FourierLaplace transform yields the density of states:

$$
g(E)=\frac{1}{i \hbar} \int_{0}^{\infty} e^{i E T / \hbar} Z(T) d T .
$$

Following the general arguments of Gutzwiller [1], one may evaluate the integrations in (6) using the stationary phase approximation, which becomes exact in the classical limit $\mathcal{R} \gg \hbar$. The semiclassical approximation of the partition function $Z(T)$ then turns into a sum over all classical periodic orbits $(p o)$ with fixed period $T$

$$
\mathcal{Z}_{s c}(T)=\sum_{p o} e^{i \mathcal{R}_{p o} / \hbar} \int \mathcal{D}[\boldsymbol{\eta}] \exp \left\{i \mathcal{R}_{p o}^{(2)}[\boldsymbol{\eta}, T] / \hbar\right\},
$$

where $\mathcal{R}_{\text {po }}$ are the principal functions (耳), evaluated now along the periodic orbits in the extended phase space. $\mathcal{R}_{\text {po }}^{(2)}[\boldsymbol{\eta}, T]$ are the second variations

$$
\mathcal{R}_{\text {po }}^{(2)}[\boldsymbol{\eta}, T]=\oint_{0}^{T}\left[\frac{1}{2} \boldsymbol{\eta} \cdot \mathcal{J} \dot{\boldsymbol{\eta}}-\mathcal{H}^{(2)}\right] d t,
$$

where $\boldsymbol{\eta}$ is the following $2(d+1)$-dimensional extended phase-space vector of small variations

$$
\boldsymbol{\eta}=(\boldsymbol{\lambda}, \nu, \boldsymbol{\rho}, \xi)=\left(\delta \mathbf{q}, \delta v \frac{2 \sqrt{\hbar S}}{\left(1+|z|^{2}\right)}, \delta \mathbf{p}, \delta u \frac{2 \sqrt{\hbar S}}{\left(1+|z|^{2}\right)}\right)
$$

and $\mathcal{J}=\left(\begin{array}{cc}0 & I \\ -I & 0\end{array}\right)$ is the $2(d+1)$-dimensional unit symplectic matrix. $\mathcal{H}^{(2)}$ is the second variation of the classical Hamiltonian, calculated along the periodic orbits:

$$
\begin{aligned}
\mathcal{H}^{(2)}= & \frac{1}{2} \sum_{i, j}\left[\lambda_{i} \lambda_{j} \frac{\partial^{2} \mathcal{H}}{\partial q_{i} \partial q_{j}}+2 \lambda_{i} \rho_{j} \frac{\partial^{2} \mathcal{H}}{\partial q_{i} \partial p_{j}}+\rho_{i} \rho_{j} \frac{\partial^{2} \mathcal{H}}{\partial p_{i} \partial p_{j}}\right] \\
+ & \frac{\kappa}{2}\left(\nu^{2}+\xi^{2}\right)\left(-u C_{1}-v C_{2}+C_{3}\right) \\
+ & \kappa \sqrt{\hbar S} \frac{\left(1+|z|^{2}\right)}{2}\left(\nu \frac{\partial \mathbf{n}}{\partial v}+\xi \frac{\partial \mathbf{n}}{\partial u}\right) \\
& \cdot \sum_{j}\left(\lambda_{j} \frac{\partial \mathbf{C}}{\partial q_{j}}+\rho_{j} \frac{\partial \mathbf{C}}{\partial p_{j}}\right) .
\end{aligned}
$$

After a stationary-phase evaluation of the Fourier integral (10), one finally obtains Gutzwiller's trace formula in the standard form [1, 19]. What is new here is that all its ingredients are obtained from the dynamics in the extended phase space: the actions $\mathcal{S}_{p o}(E)=\mathcal{R}_{p o}+E T_{p o}$ (at fixed energy $E$ ) and the periods $T_{p o}=d S_{p o} / d E$ of the periodic orbits, the Maslov indices $\sigma_{p o}$ for which Sugita has given general formulae [19], and the monodromy matrix $\mathcal{M}_{p o}$ defined by $\boldsymbol{\eta}\left(T_{p o}\right)=\mathcal{M}_{p o} \boldsymbol{\eta}(0)$ in terms of the solutions of the linearized equations of motion $\dot{\boldsymbol{\eta}}=\mathcal{J} \partial \mathcal{H}^{(2)} / \partial \boldsymbol{\eta}$. We refer to a forthcoming extended paper [20] for the details of our calculations, where we also show that in the weak-coupling limit we obtain the same results as Bolte and Keppeler [8]. 
We shall presently illustrate our method by applying it to a simple model Hamiltonian describing a twodimensional electron gas $(S=1 / 2)$ in a semiconductor heterostructure, laterally confined to a quantum dot, including a spin-orbit interaction of Rashba type [21]

$$
\widehat{H}=\frac{\left(\hat{p}_{x}^{2}+\hat{p}_{y}^{2}\right)}{2 m^{*}}+V(x, y)+\kappa \hbar\left(\sigma_{y} \hat{p}_{x}-\sigma_{x} \hat{p}_{y}\right) .
$$

Here $m^{*}$ is the effective mass of the electron, $\sigma_{i}$ are the Pauli matrices, and the lateral confining potential $V(x, y)$ is approximated as a deformed harmonic oscillator

$$
V(x, y)=m^{*}\left(\omega_{x}^{2} x^{2}+\omega_{y}^{2} y^{2}\right) / 2 .
$$

We use $\omega_{x}=1.56 \omega_{0}, \omega_{y}=1.23 \omega_{0}$, and units such that $\hbar=m^{*}=\omega_{0}=1$ and that $E$ and $\kappa$ become dimensionless. The spin-orbit coupling strength $\kappa$ in (14) depends on the band structure 22]. E.g., for a InGaAs-InAlAs quantum dot with $\sim 100$ confined electrons one would obtain a value of $\kappa \sim 0.25$. We investigate in the following a situation where $\kappa$ is large enough for the spin dynamics to affect the orbital motion. For the examples below we have chosen $\kappa=0.67$. The equations of motion (9) were solved numerically.

For a large range of parameters with $0<\kappa \lesssim 0.75$, we find the following periodic solutions of Eq. (9): 1) two pairs of adiabatic orbits $\mathrm{A}_{x}^{ \pm}$and $\mathrm{A}_{y}^{ \pm}$, librating along the $x$ and $y$ axes with fully polarized spin $n_{y}= \pm 1$ and $n_{x}= \pm 1$, respectively; 2 ) two pairs of diabatic orbits, $\mathrm{D}_{x 1}^{ \pm}$ and $\mathrm{D}_{x 2}^{ \pm}$, oscillating around $\mathrm{A}_{x}^{ \pm}$with $n_{y} \sim 0$; and 3) two pairs of diabatic orbits, $\mathrm{D}_{y 1}^{ \pm}$and $\mathrm{D}_{y 2}^{ \pm}$, oscillating around $\mathrm{A}_{y}^{ \pm}$with $n_{x} \sim 0$. The superscripts $( \pm)$ of the diabatic orbits denote their senses of rotation. The stabilities of these orbits will be discussed elsewhere [20]. For stronger couplings $\kappa \gtrsim 0.75$ and for energies $E \lesssim 8$, new orbits bifurcate from the A and D orbits. In these situations, the stability amplitudes will have to be regularized by suitable uniform approximations [23].

The diabatic orbits obtained in our approach reflect the explicit coupling of the spin and orbital degrees of motion. Such orbits have not been discussed in earlier semiclassical methods. The adiabatic orbits with "frozen spin" correspond to those discussed in [6,7,10] where they could not be used semiclassically, though, because of the mode conversion occurring at their turning points. We repeat that we do not encounter the mode-conversion problem here, and that the stability amplitudes of all orbits can be readily calculated numerically.

In Fig. 1 we present the $(x, y)$ shapes of the orbits $\mathrm{A}_{x}^{+}$, $\mathrm{D}_{x 1}^{+}$, and $\mathrm{D}_{x 2}^{+}$(left panels), and the time dependence of their spin components $n_{x}, n_{y}$, and $n_{z}$ over one period (right panels), all evaluated at $E=60$. (The orbits $\mathrm{A}_{y}^{ \pm}$, $\mathrm{D}_{y 1}^{ \pm}$, and $\mathrm{D}_{y 2}^{ \pm}$have analogous shapes concentrated along the $y$ axis, and the behavior of their $n_{x}$ and $n_{y}$ components is reversed.) We see that along the diabatic orbits $\mathrm{D}_{x 1}^{+}$and $\mathrm{D}_{x 2}^{+}$, the spin rotates mainly near the $\left(n_{x}, n_{z}\right)$ plane (with $n_{y} \sim 0$ ) in a non-uniform way. The diabatic
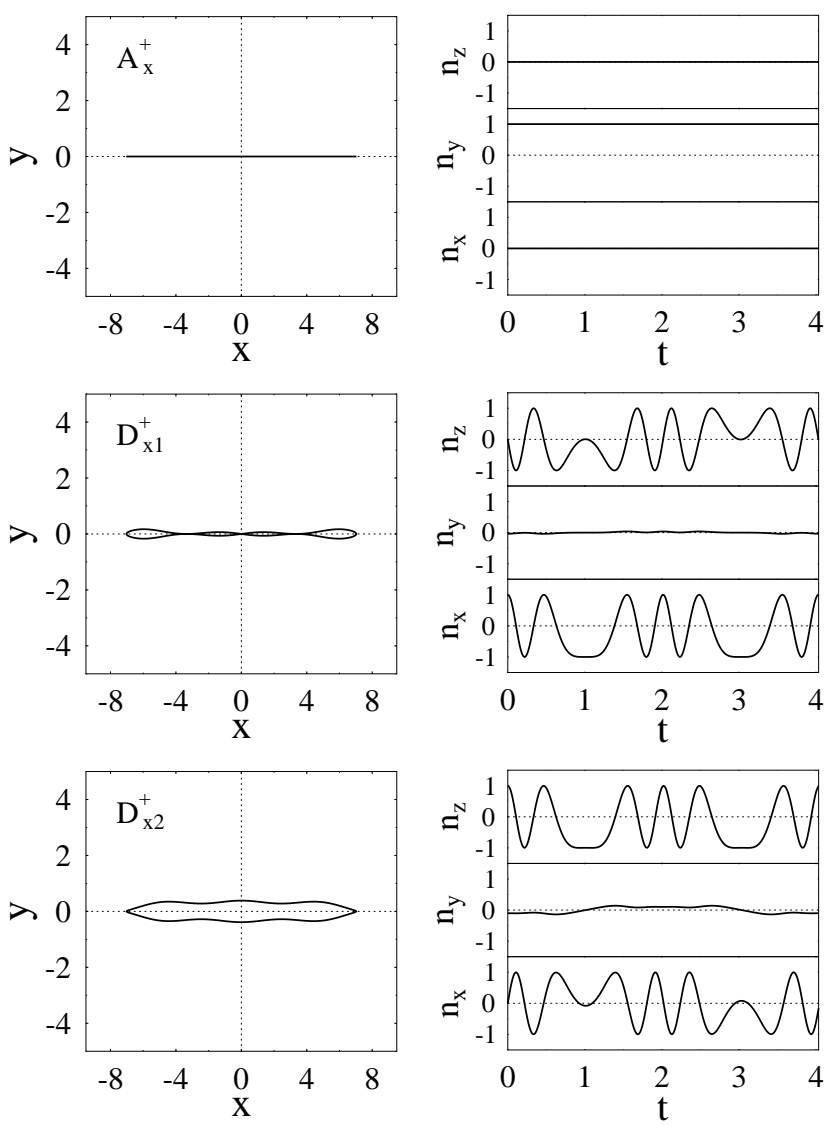

FIG. 1. Periodic orbits in the 2-dimensional harmonic oscillator with Rashba spin-orbit interaction (see text for parameters). Left panels: orbits in the $(x, y)$ plane. Right panels: Spin components $n_{x}, n_{y}$, and $n_{z}$ versus time. From top to bottom: Adiabatic orbit $\mathrm{A}_{x}^{+}$along $x$ axis with polarized spin in $y$ direction, and diabatic orbits $\mathrm{D}_{x 1}^{+}$and $\mathrm{D}_{x 2}^{+}$oscillating around the $x$ axis with spin rotating near the $\left(n_{x}, n_{z}\right)$ plane.

spin-flip hypothesis of [7] has thus been replaced here by the more sophisticated spin dynamics obtained from the coupled equations of motion (9).

In Fig. 2 we show the oscillating part of the density of states $\delta g(E)$, obtained quantum-mechanically (solid line) using exact diagonalization of the Hamiltonian (14), and semiclassically (dashed line) using Gutzwiller's trace formula. Since the periodic orbit sum generally does not converge in systems with mixed classical dynamics, we have convoluted it with a normalized Gaussian, $\exp \left\{-(E / \gamma)^{2}\right\} / \gamma \sqrt{\pi}$. This brings the sum to convergence [24, whereby only the orbits with shortest periods contribute, and reflects the prominent gross-shell structure of the quantum spectrum. We have used $\gamma=0.6$ where it was sufficient to include the above twelve primitive periodic orbits. We observe a rather good agreement between the semiclassical and quantum-mechanical results. The regular beat-like structure comes about through the interference of the six types of classical orbits which all have frequencies close to either $\omega_{x}$ or $\omega_{y}$. 


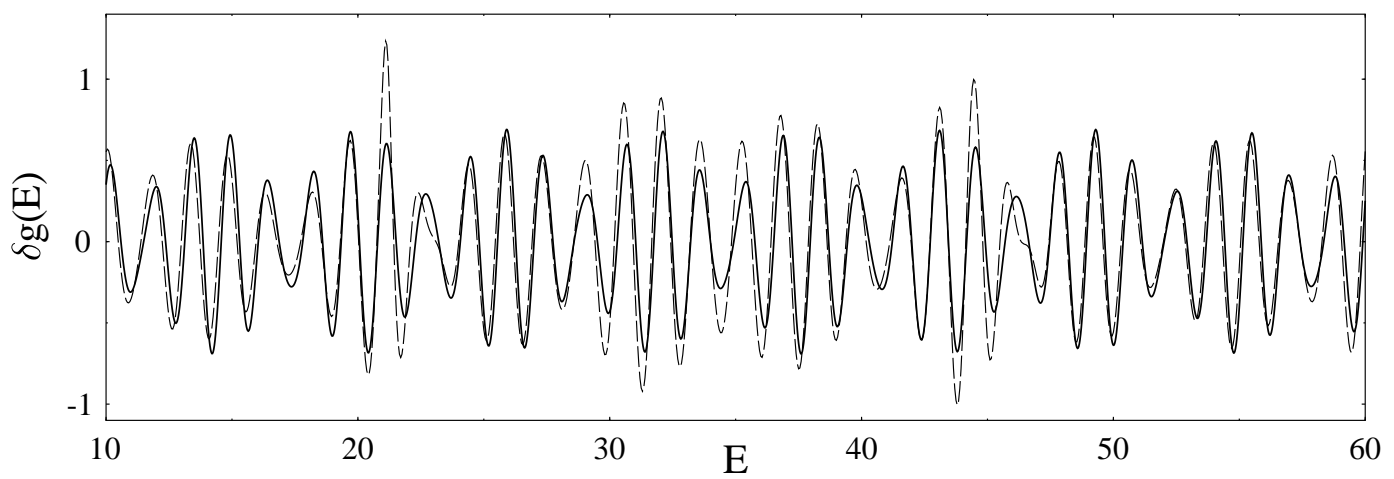

FIG. 2. Oscillating part of the coarse-grained density of states (Gaussian averaging parameter $\gamma=0.6$ ) versus energy for the same system as in Fig. 1. Solid line: quantum-mechanical result, dashed line: semiclassical result using the 12 primitive orbits.

In summary, we have presented a novel approach to include a non-adiabatic coupling of spin and orbital dynamics into the semiclassical theory of periodic orbits. Our approach overcomes some of the difficulties with earlier approaches, in particular the restriction to purely adiabatic spin motion and the problem of mode conversion. For the simple model of a two-dimensional quantum dot with Rashba spin-orbit interaction, we obtain a satisfactory semiclassical description of the coarse-grained density of states. To study its experimental implications, we are in the process of calculating semiclassically the conductance of this system in response to an external magnetic field [20].

We should point out that there exists a subtle problem connected with the fact that the measure $\mathcal{D}[\boldsymbol{\eta}]$ of the path integral over the extended phase-space variations $\boldsymbol{\eta}$ in (11) has the proper normalization only in the large-spin limit. For small spin, like $S=1 / 2$, this calls for an appropriate renormalization scheme 25]. Although the different renormalizations needed for pure spatial motion [26] and pure spin motion [13] are known, a valid scheme for the combined spin-orbit dynamics under investigation here is yet to be found. It might lead to extra phase corrections in the semiclassical expressions, as discussed in [27], and thereby affect the numerical results to some extent.

We acknowledge helpful and critical discussions with O. Zaitsev and encouraging comments by K. Richter.

* Work supported by Deutsche Forschungsgemeinschaft.

[1] M. C. Gutzwiller, J. Math. Phys. 12, 343 (1971).

[2] M. C. Gutzwiller: Chaos in Classical and Quantum Mechanics (Springer, New York, 1990); K.-F. Berggren and S. Åberg (Eds.): Quantum Chaos Y2K, Proceedings of Nobel Symposium 116, Physica Scripta Vol. T90 (2001).

[3] M. Brack and R. K. Bhaduri: Semiclassical Physics, Frontiers in Physics Vol. 96 (Addison-Wesley, Reading, USA, 1997).

[4] M. Oestreich, Nature 402, 735 (1999); S. Das Sarma, J. Fabian, X. Hu, and I.Zutic, IEEE Trans. Magn. 36, 2821 (2000).

[5] S. Datta and B. Das, App. Phys. Lett. 56, 665 (1990); see also S. Das Sarma, Am. Sci. 89, 516 (2001).

[6] R. G. Littlejohn and W. G. Flynn, Phys. Rev. A 44, 5239 (1991); Phys. Rev. A 45, 7697 (1992).

[7] H. Frisk and T. Guhr, Ann. Phys. (N.Y.) 221, 229 (1993).

[8] J. Bolte and S. Keppeler, Phys. Rev. Lett. 81, 1987 (1998); Ann. Phys. (N.Y.) 274, 125 (1999).

[9] S. Keppeler and R. Winkler, Phys. Rev. Lett. 88, 046401 (2002).

[10] Ch. Amann and M. Brack, J. Phys. A 35, 6009 (2002).

[11] C.-M. Hu et al., Phys. Rev. B 60, 7736 (1999); T. Matsuyama et al., Phys. Rev. B 61, 15588 (2000).

[12] see, e.g., A. Bohr and B. R. Mottelson: Nuclear Structure, Vol. I (W. A. Benjamin, New York, 1969).

[13] J. R. Klauder, Phys. Rev. D 19, 2349 (1979).

[14] H. Kuratsuji and T. Suzuki, J. Math. Phys. 21, 472 (1980); H. Kuratsuji and Y. Mizobuchi, J. Math. Phys. 22, 757 (1981).

[15] A. M. Perelomov, Commun. Math. Phys. 26, 222 (1972).

[16] E. Kochetov, J. Math. Phys. 36, 4667 (1995).

[17] V. Smirnov, J. Phys. A 32, 1285 (1999).

[18] A. Alscher and H. Grabert, Eur. Phys. J. D 14, 127 (2001).

[19] A. Sugita, Ann. Phys. (N.Y.) 288, 277 (2001).

[20] M. Pletyukhov, O. Zaitsev, and M. Brack, to be published.

[21] Y. Bychkov and E. Rashba, J. Phys. C 17, 6039 (1984), and earlier references quoted therein.

[22] see, e.g., T. Darnhofer, M Suhrke, and U. Rössler, Europhys. Lett. 35591 (1996).

[23] M. Sieber and H. Schomerus, J. Phys. A 31, 165 (1998), and earlier references.

[24] M. Sieber and F. Steiner, Phys. Rev. Lett. 67, 1941 (1991).

[25] M. Miettinen, J. Math. Phys. 37, 3141 (1996).

[26] H.G. Solari, J. Math. Phys. 28, 1097 (1987).

[27] M. Stone, K.-S. Park, and A. Garg, J. Math. Phys. 41, 8025 (2000). 